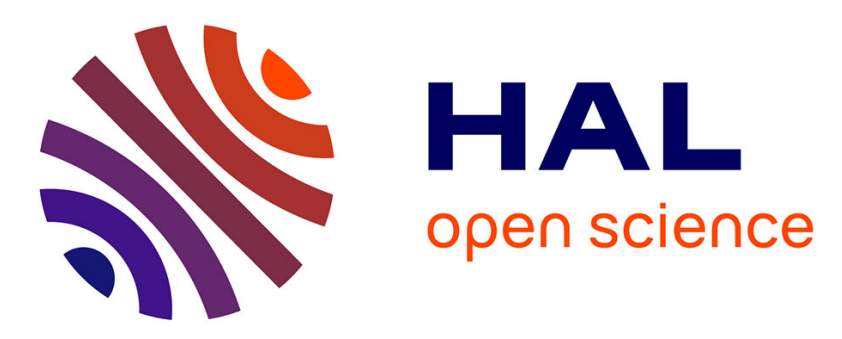

\title{
Sensing One Nanometer Over Ten Centimeters: A Microencoded Target for Visual In-Plane Position Measurement
}

\author{
Antoine André, Patrick Sandoz, Benjamin Mauze, Maxime Jacquot, \\ Guillaume Laurent
}

\section{To cite this version:}

Antoine André, Patrick Sandoz, Benjamin Mauze, Maxime Jacquot, Guillaume Laurent. Sensing One Nanometer Over Ten Centimeters: A Microencoded Target for Visual In-Plane Position Measurement. IEEE/ASME Transactions on Mechatronics, 2020, 25 (3), pp.1193 - 1201. hal-03423034

\section{HAL Id: hal-03423034 https://hal.science/hal-03423034}

Submitted on 9 Nov 2021

HAL is a multi-disciplinary open access archive for the deposit and dissemination of scientific research documents, whether they are published or not. The documents may come from teaching and research institutions in France or abroad, or from public or private research centers.
L'archive ouverte pluridisciplinaire HAL, est destinée au dépôt et à la diffusion de documents scientifiques de niveau recherche, publiés ou non, émanant des établissements d'enseignement et de recherche français ou étrangers, des laboratoires publics ou privés. 


\title{
Sensing One Nanometer over Ten Centimeters: A Micro-Encoded Target for Visual In-Plane Position Measurement
}

\author{
Antoine N. André, Patrick Sandoz, Benjamin Mauzé, Maxime Jacquot, Guillaume J. Laurent
}

\begin{abstract}
At the nanoscale, accurate control and position sensing are recurrent issues conditioning advances of technologies and instruments in diverse fields of application. Recent advances were reported that combine optical microscopy and computer vision for non-contact and multi-axis position sensing of end-effectors with a high accuracy. This paper contributes to this domain by introducing a micro-encoded target aimed to high accurate $(x, y, \theta)$ measurements over an extended range. The micro-encoded target is designed to allow linear phase measurements, leading to high accuracy in position sensing, and unambiguous binary encoding leading to absolute position determination. In this way, ultimate performances and allowed measurement ranges are independent of each other and a current zone of observation can be registered with respect to the whole encoded area with an extra-large range-to-resolution ratio. Experiments reported in this paper demonstrate a precision below one nanometer in lateral position and of $4 \cdot 10^{-6} \mathrm{rad}$. in rotation with an encoded target of more than $11 \times 11 \mathrm{~cm}^{2}$. The device demonstrated can be either inserted into experimental setups to allow accurate position control or used to calibrate in-plane displacements of one to three degrees of freedom actuators.
\end{abstract}

\section{Index Terms}

Nano-positioning, Position encoding, Pseudo-random binary sequences, Phase measurements, Computer vision

\section{INTRODUCTION}

Nanopositioning is basic to all areas of nanotechnology from manufacturing to metrology. Some applications like scanningprobe microscopy and nanoindentation only need very small movement controlled with a high resolution, while others such as wafer inspection and microrobotics require a high degree of accuracy over long ranges in several directions. As anticipated by NIST in 2008, emerging nanotechnology industries will require revolutionary metrology with higher resolution and accuracy than had previously been envisioned [1].

At the macroscale, many instruments allow to measure position along several directions with high range-to-resolution ratios. For instance, coordinate measuring machines (CMM) are able to measure the geometry of physical objects by sensing discrete points along the $\mathrm{X}, \mathrm{Y}$, and $\mathrm{Z}$ axes, typically with micrometer precision over a meter. Laser trackers (LT) used to calibrate robots or to measure large objects up to tens of meters can achieve pose estimation with micrometers resolution by determining the positions of optical targets held against those objects. The range-to-resolution ratio of both instruments are generally better than $10^{7}$ (see Fig. 1). Even at a larger scale, the global positioning system (GPS) has a relative high precision, pinpointing to within 30 centimeters over the earth's circumference leading to range-to-resolution ratio over $10^{8}$.

At the nanoscale, interferometers (IF) are considered and used as reference measuring systems. It's one of the only solutions to reach nanometer precisions over meter ranges but recent works involving optical microscopy and image processing have pushed the limits of multi-axis pose estimation at the millimeter, micrometer and nanometer scales [2]-[4]. Although optical microscopy is a mature field, it continues to see exciting new developments. For instance, Zhao et al. proposed in 2019 an in-plane $\mathrm{XY \Theta}$ pose estimation method that achieves a resolution of $9.35 \mathrm{~nm}$ by analyzing the intersection of regular sets of straight and circular lines micro-machined on a polar micro-structure [5].

For the purpose of in-plane $\mathrm{XY} \Theta$ pose estimation, visual measuring methods can be distinguished between feature-based and area-based methods. First methods utilize local features of the image, such as corners or edges, to retrieve displacements and can reach sub-micrometer resolution. Indeed, although several works have shown that local methods achieve resolution so far as one tenth of a pixel, the resolution in optical microscopy is limited by the diffraction of the light leading to ultimate resolution near $10 \mathrm{~nm}[6]-[8]$. Using a scanning electron microscope could be considered to improve the resolution of the image but at the detriment of the measurement range [9]. As can be seen on Fig. 11, range-to-resolution ratios of featured-based methods are below $10^{5}$. In addition, featured-based methods are sensitive to image sharpness and signal-to-noise ratio (SNR).

On the other hand, area-based methods consider a large area of the image and this results in averaging the local effects of sharpness and SNR. They are based on the existence of an image texture, either naturally present in the scene of interest or specifically inserted. Most of these methods are variations of the original correlation method and exploit the redundancy of spatial information to achieve resolution better than one thousandth of a pixel [10]-[13]. To increase the SNR and to

The authors are with FEMTO-ST Institute, Univ. Bourgogne Franche-Comté, UMR CNRS 6174, Besançon, France \{antoine.andre, patrick.sandoz, benjamin.mauze, maxime.jacquot, guillaume.laurent\} @femto-st.fr 


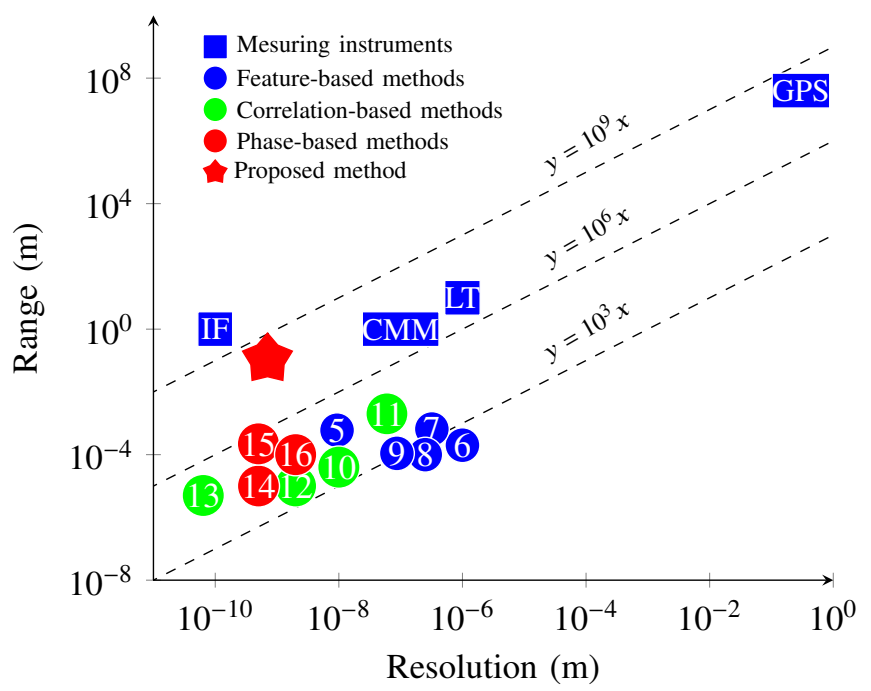

Fig. 1. State of the art comparison in term of range-to-resolution performances between standard measuring instruments and vision-based methods used in microscopy.

get calibrated measurements, some authors propose to use a periodic pattern attached to the object of interest. For instance, Sugiura implemented a Moiré fringe method to sense a micro probe position that reaches a resolution of $42 \mathrm{~nm}$ on a limited range [17]. Yamahata performed a differential phase measurement in the Fourier spectrum to characterize the displacement with a sub-nanometer resolution of a MEMS actuator [14].

However, phase-based methods are limited to displacements shorter than the pattern period. This issue of extending the allowed range of phase-based methods beyond the period has been addressed in several ways. The choice of an extended periodic frame makes the allowed displacement range independent of the field of observation of the camera but requires some kind of additional codification to remove ambiguities of an entire number of periods. A simple solution, inherited from interferometry, consists in using two fringe sets with different periods. In 2016, we proposed a 2D-interleaved twin-scale pattern and demonstrated a experimental resolution below $0.5 \mathrm{~nm}$ over an unambiguous range of $221 \mu \mathrm{m}$ using direct phase measurements [15]. Another common solution to enlarge the range is to combine a binary encoding to the periodicity. For that purpose, the use of pseudo-random binary sequences obtained by linear feedback shift register (LFSR) is very convenient [18]. The unique property of LFSR sequences is that every binary word of $n$ bits shares $n-1$ bits with its immediate neighbors. Therefore, whatever the number of bits chosen, the LFSR sequence can be materialized on a single track position encoder. This technique was used by Stevenson and Jordan two decades ago with a Manchester code to design 2D position encoders [19]. The Manchester code was also used by Masa et al. in addition to regular gratings for direct phase measurement in their 2D absolute encoder [20]. Kim et al. use a similar method with a different bit-coding technique and achieved a $15 \mathrm{~nm}$ resolution from a smallest feature size and elementary period of $4 \mu \mathrm{m}$ and $32 \mu \mathrm{m}$ respectively [21]. However, in Stevenson, Masa and Kim approaches, the shifts induced for differentiating 0 and 1 bit values result in non-linearities detrimental to resolution.

This paper proposes an absolute binary encoding method preserving the phase distribution of the periodic frame, and thus avoiding non-linearities, with a significant improvement of performances achieved. The proposed micro-encoded target and its associated processing is intended for $\mathrm{XY} \Theta$ sensing over a range up to $11 \mathrm{~cm}$ with a sub-nanometer resolution.

Section II describes the design of the micro-encoded target and emphasizes on its phase preservation properties. Section III presents the image processing developed for in-plane position reconstruction and estimates the ultimate method performances through computer simulations. Section IV presents experimental results obtained and section V concludes on the performances demonstrated using the micro-encoded target.

\section{Micro-EnCODED TARGET DESIGN}

Our initial aim was to demonstrate a $10^{8}$ range-to-resolution ratio by achieving sub-nanometer precision combined with a $10 \mathrm{~cm}$ measurement range by means of a micro-encoded target. For this purpose and in regard to bibliography, the target has been designed to fulfill two complementary requirements: $i)$ to present a periodic frame suitable for high-resolution phase measurements, ii) to allow the unambiguous identification of the consecutive periods to encode a range of $10 \mathrm{~cm}$. A supplementary practical requirement is that the smallest features must be detected without requiring high magnification lenses that would reduce the available work-space because of shorter working distances.

The periodic frame designed is based on linear shift register sequences and has been thought in order to preserve phase continuity and linearity. Fig 2 compares the code chosen with respect to the Manchester code, notably used by Stevenson and Jordan, and to the phase encoded scale of Kim et al [19], [21]. The code that we used appears in Fig2]e and is based on a 


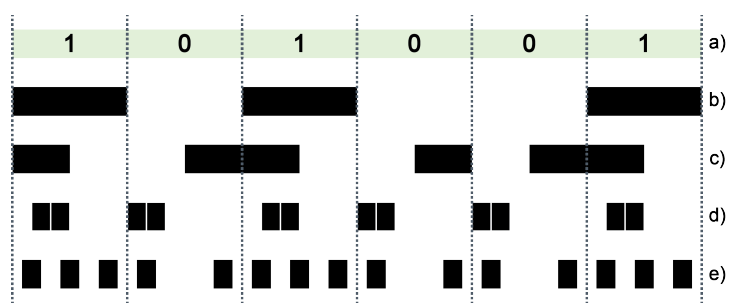

Fig. 2. Diverse methods for bit encoding: a) binary sequence; b) black and white code; c) Manchester code [19]; d) Kim et al.'s code [21]; e) phase preserving code proposed in this paper.

TABLE I

CONCEPTION TABLE FOR TARGET SIZE CHOICE WITH A VARYING PERIOD SIZE ( $\lambda$ : PHYSICAL PERIOD.)

\begin{tabular}{c|c|c|c|c}
$\begin{array}{c}\text { order } n \text { for the } \\
\text { LFSR generation }\end{array}$ & Code size & \multicolumn{3}{|c}{ Resulting target size } \\
& & $\lambda=3 \mu \mathrm{m}$ & $\lambda=9 \mu \mathrm{m}$ & $\lambda=25 \mu \mathrm{m}$ \\
\hline 3 bits & 24 & $72 \mu \mathrm{m}$ & $216 \mu \mathrm{m}$ & $600 \mu \mathrm{m}$ \\
6 bits & 192 & $1.15 \mathrm{~mm}$ & $1.7 \mathrm{~mm}$ & $4.8 \mathrm{~mm}$ \\
8 bits & 768 & $2.3 \mathrm{~mm}$ & $6.9 \mathrm{~mm}$ & $19.2 \mathrm{~mm}$ \\
10 bits & 3072 & $0.92 \mathrm{~cm}$ & $2.76 \mathrm{~cm}$ & $7.68 \mathrm{~cm}$ \\
12 bits & 12288 & $3.68 \mathrm{~cm}$ & $11.06 \mathrm{~cm}$ & $30.72 \mathrm{~cm}$
\end{tabular}

regular distribution of stripes that are grouped by sets of three periods. The binary value ' 1 ' is made of three present periods whereas, for the binary value ' 0 ', the central period is missing. This choice maintains a high density in the frequency carrier without introducing phase non-linearities. Conversely, in Fig2 $2 \mathrm{c}$ and Fig 2 d, the black areas are shifted in accordance to the binary value and this alters the local phase and introduces non-linearities.

Following the proposed scheme, the encoded range depends on the number of bits and on the period size as shown in Table I. The final trade-off chosen is a period of $9 \mu \mathrm{m}$ with a 12 bit encoding that leads to an encoded length of more than $11 \mathrm{~cm}$ and compatible with a $10 \times$ imaging lens.

The 2D code is obtained by reproducing the same code along the perpendicular direction. To avoid rotation ambiguities, we broke the $\pi / 2$ symmetry by removing on square at one corner. Then each intersection of a binary line with a binary column results in a coding cell of 9 spots as depicted in Fig $3 \mathrm{a}$ and $3 \mathrm{~b}$. Furthermore, in the final LFSR sequence, the word of value $2^{n}-1$ is removed in order to have at least one zero in every set of $n$ bits since the missing lines associated with the value 0 serves as embedded clock to discriminate coding cell frontiers.

In practice, the micro-encoded target has been realized in clean room with a commercial mask generator (Heidelberg DWL200) of which X,Y positions, displacements and alignment are continuously controlled by a set of three interferometers. The target obtained corresponds to the designed distribution of holes etched on a thin chromium layer deposited on a $12.7 \times$ $12.7 \mathrm{~cm}^{2}$ piece of glass with a thickness of $3 \mathrm{~m}$. Fig 4 a presents a partial view of the micro-encoded target realized as recorded experimentally with the setup presented in section IV] The size of the micro-encoded target can be reduced by dicing to fit various setup needs without performance alterations except the allowed range of measurement.

To summarize, the measurement principle consists in retrieving the coordinate $(x, y)$ on the encoded target of the local image recorded with the camera as well as its orientation $\theta$ with respect to the lines and columns of the target. In regard to the phase measurements, the positions $(x, y)$ correspond to absolute phases $\left(\phi_{x}, \phi_{y}\right)$ respectively equal to $2 \pi x / \lambda$ and $2 \pi y / \lambda$ with $\lambda$ the physical period of the encoded target.

(a)

$$
\left[\begin{array}{ccc}
O F F & x_{b i t} & O N \\
y_{b i t} & x_{b i t} \cdot y_{b i t} & y_{b i t} \\
O N & x_{b i t} & O N
\end{array}\right]
$$

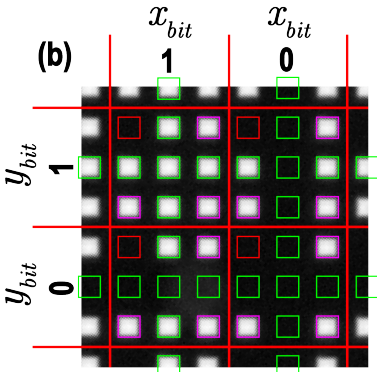

Fig. 3. Definition and instances of coding cells, (a) Coding cell configuration: Corners are independent of binary values with one corner switched to Off to remove $\pi / 2$ ambiguities. Central line and column are set On or Off in accordance with the current binary value. (b) Coding cells obtained for the 4 ( $x, y$ ) binary combinations and for a single angular direction. Colored squares discriminate coding spots from non-coding ones. 


\section{Pose ESTIMATION AND COMPUTER CODE VALIDATION}

\section{A. Image processing}

Once an image of a small-sized zone of the micro-encoded target has been recorded, the aim of image processing is to determine with the best accuracy the localization of this zone within the whole encoded area of $11 \times 11 \mathrm{~cm}^{2}$. The reconstruction of the $(x, y, \theta)$ coordinates relies on phase computations and digital decoding following several sequential steps as described in the following and in Fig. 4

The first processing step consists in retrieving the phase related to the two sets of straight lines associated to the perpendicular directions of the target. This demultiplexing is based on band-pass filtering applied to the Fourier transform of the recorded image. The different spectral lobes visible in Fig. 4. b correspond to the spatial frequencies of the recorded image presented in Fig. 4. a. Since the initial image is real, the Fourier spectrum obtained is symmetrical with each lobe associated to a complex conjugate at the diagonal of the spectrum center. Then, a first maximum $f_{1}$ is detected in the spectrum and a hyper-Gaussian filter centered on this particular frequency is applied. By selecting a single lobe, we break the symmetry and, after inverse Fourier transform, this results in a complex representation of the line associated to lobe $f_{1}$. The wrapped phase distribution is then directed given by the argument of the complex data provided by the inverse Fourier transform as depicted in Fig. $4 . c_{1}$ for a sub-area. The same procedure is applied similarly to the frequency component $f_{2}$ associated to the perpendicular direction (Fig. $4 c_{2}$ ). More details on this phase processing can be found in [22].

The wrapped phases of Fig. 4. $c_{1}$ and 4. $c_{2}$ are used in different ways for complementary fine and coarse position determinations. The fine position determination consists in localizing the straight lines with respect to the pixel frame of the image independently from the LFSR absolute encoding. From the target design, we know that the wrapped phase of Fig. $4 . c$ corresponds to a continuous and linear distribution of phase associated to the set of parallel straight lines of each direction. Therefore, the wrapped phase can be unwrapped to obtain a phase plane (not shown, see [22]). This operation corresponds to increment the order of the straight lines at each $2 \pi$ jump to fit the continuous distribution of parallel lines. The phase planes obtained are fitted with a least square equation that allows the determination of the phase related to the central pixel of the image with a high resolution as well as the orientation of the lines represented by the red arrows in Fig. $4 . c_{1}$ and $4 c_{2}$.

At this stage, the order of the lines is not registered with respect to the whole target but is relative to the first image point considered in the unwrapping process. However, the sub-period phase obtained through this procedure is fully representative of the position of the lines with respect to the pixel frame of the camera. The equations of the phase planes retrieved can be expressed as given in Eq. 1 and 2

$$
\begin{gathered}
\phi_{1}(i, j)=\frac{2 \pi i \cos \theta}{P}+\frac{2 \pi j \sin \theta}{P}+\phi_{x / y}+2 k_{1} \pi \\
\phi_{2}(i, j)=\frac{2 \pi i \cos (\theta+\pi / 2)}{P}+\frac{2 \pi j \sin (\theta+\pi / 2)}{P}+\phi_{y / x}+2 k_{2} \pi
\end{gathered}
$$

with $P$ the line period in pixels. $k_{1}, k_{2}$ stand for unknown entire numbers of lines/columns separating the unwrapped phase planes obtained at this stage from the expected absolute phase planes registered with respect to the encoded target origin. The correct association of the phase planes $\phi_{1}$ and $\phi_{2}$ with the target directions $x$ and $y$, and with the absolute phases $\phi_{x}$ and $\phi_{y}$, will be determined later from the position of the missing corner of the coding cells. The period $P$ in pixels is related to the physical distance $\lambda$ between lines and columns by: $P=\lambda \cdot \mathrm{Mag} / \mathrm{PixelSize}$, where Mag is the optical magnification of the imaging system and PixelSize the physical size of pixels on the image sensor. In Eq. 1 , the plane $\operatorname{coefficients~} 2 \pi \cos \theta / P$ and $2 \pi \sin \theta / P$ correspond to the coordinates of the spectral lobe $f_{1}$ in Fig. $4 . b$ while the lobe $f_{2}$ is located at coordinates $2 \pi \cos (\theta+\pi / 2) / P$ and $2 \pi \sin (\theta+\pi / 2) / P$. Since these phase plane coefficients differ only by the sine and cosine functions of $\theta$, the orientation of the lines and columns within the image is directly obtained through an inverse tangent operation.

The aim of the coarse pose estimation step consists in determining the right line order through the decoding of the LFSR sequence present in the image. This is necessary to adjust the relative phase planes with the correct number of periods; i.e. to remove the $2 k_{1} \pi$ and $2 k_{2} \pi$ terms in Eq. 1 and 2, and thus register absolutely the zone observed within the entire target. For this purpose, among each set of three lines and columns, the one representative of the bit value as a function of its presence or absence has to be determined. This identification is based on the comparison of the relative intensities of consecutive lines and columns. The absent lines and columns associated to the 0 bit value lead to a minimum that allows the identification of the coding ones. Then the mean intensity of each coding line and column is compared to the background intensity to determine the associated binary value. In this process, the wrapped phase is used to localize the position of the white spots, of which phase is close to 0 , with respect to the dark background of which phase is close to $\pm \pi$. This phase-guided local contrast determination makes the binary decoding very robust, notably against possible illumination non-uniformity across the field of observation. The missing spot aimed to remove $\pi / 2$ angular ambiguities is identified in the same way as shown in Fig. $4 d_{1}$ and $4 d_{2}$. Finally, the unwrapped phase maps can be adjusted with the correct number of periods to be fully representative of the position of the observed zone in regard to the whole target as represented in Fig. 4. $e$. The success of this absolute registration procedure requires the observation of at least one word of 12 bits; i.e. about 40 lines and columns of spots. However, these 40 lines and 


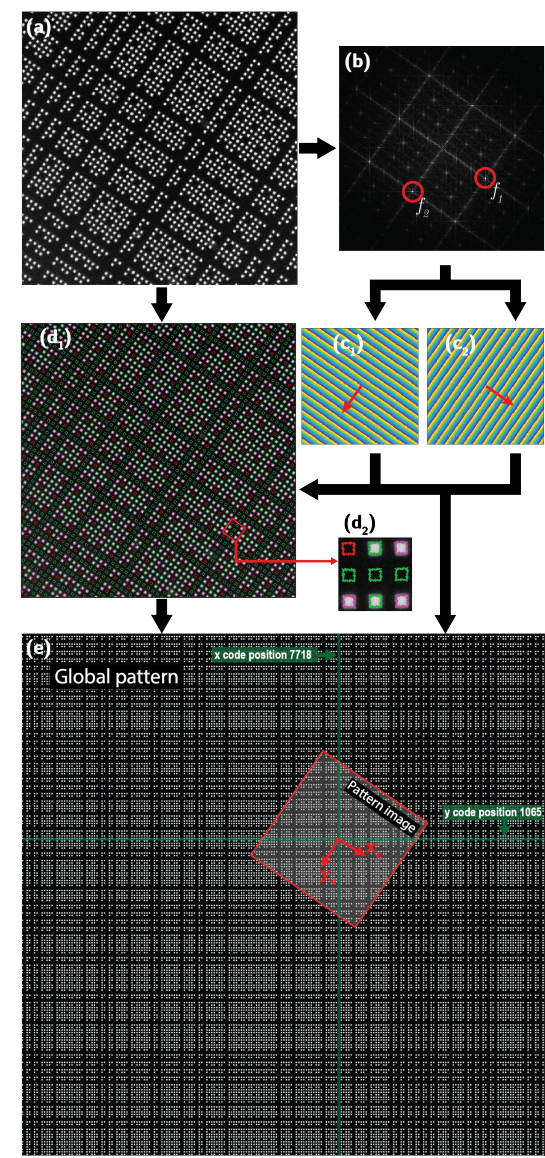

Fig. 4. Pose estimation process, (a) acquired target image, (b) Modulus of the $2 \mathrm{D}$ discrete Fourier transform. Circles exhibit the two frequency peaks of the two directions of the target image. The application of a Gaussian filter and inverse Fourier transform, result in the wrapped phase maps $\left(c_{1}\right)$ and $\left(c_{2}\right)$ representative of the line position with respect to the image pixel frame. $\left(d_{1}\right)$ presents the binary decoding necessary to identify the coding cells and the $2 \mathrm{D}$ LFSR sequences. $\left(d_{2}\right)$ zooms on a single coding cell with colored squares discriminating corners from coding line and column. As a result, (e) shows the fine and absolute registration of the current image within the encoded target.

columns are not required to be present throughout the whole image since their binary value can be identified even when only several periods are visible in an image corner (Fig. $4 d_{1}$ ).

In this image processing, the knowledge that the physical period of the target is equal to $9 \mu \mathrm{m}$ allows the conversion of phase data into actual positions and distances on the encoded target without any requirement for vision system calibration. The reliability of this procedure will be discussed in the result section.

A video illustrating the decoding process on a rotation example can be found as supplementary material.

This process, coded in $\mathrm{C}++$ has the advantage to be as close as theoretical results can give. Every function programmed comes with a test function that ensures the final position computed is equal (minus the numerical noise) to what theory predicts. Current $C++$ software offers a $5 \mathrm{~Hz}$ measurement rate on $1024 \times 1024$ pixel images and $30 \mathrm{~Hz}$ with $512 \times 512$ pixel images with a quantification depth of 12 bits. In terms of complexity, the process requires one direct 2D Fourier transform, two inverse ones and some additional $O(n)$ computations for the decoding (with $n$ the total number of pixels). The method is easily shown to have the same $O(n \log n)$ complexity as the Fourier transforms.

\section{B. Estimation of process performances}

Computer simulations were carried out in order to determine the level of errors induced by round-off of the numerical computations in the absence of experimental disturbances such as spurious environmental displacements and imaging noise. For that purpose, we generated synthesis images of the target for a set of 200 random $(x, y, \theta)$ positions and compared the reconstructed positions with the expected ones. We chose an arbitrary period of 20.123 pixels which is close to the one obtained experimentally from the actual encoded target with a physical period of $9 \mu \mathrm{m}$. This choice allows the conversion of numerical errors in pixels into actual distances representative of the uncertainty level. Typical residual errors observed are represented in Fig. 5 $a_{1}, b_{1}, c_{1}$ for $x, y, \theta$ measures respectively. We note that the shape of the histograms of the errors values (Fig $5 a_{2}, b_{2}, c_{2}$ ) is close to a Gaussian distribution of residual errors. Fig. $5 d_{1}$ and $d_{2}$ show the location of the explored positions and angles with respect to the whole target and the $]-\pi, \pi]$ interval respectively. The standard deviations of the 

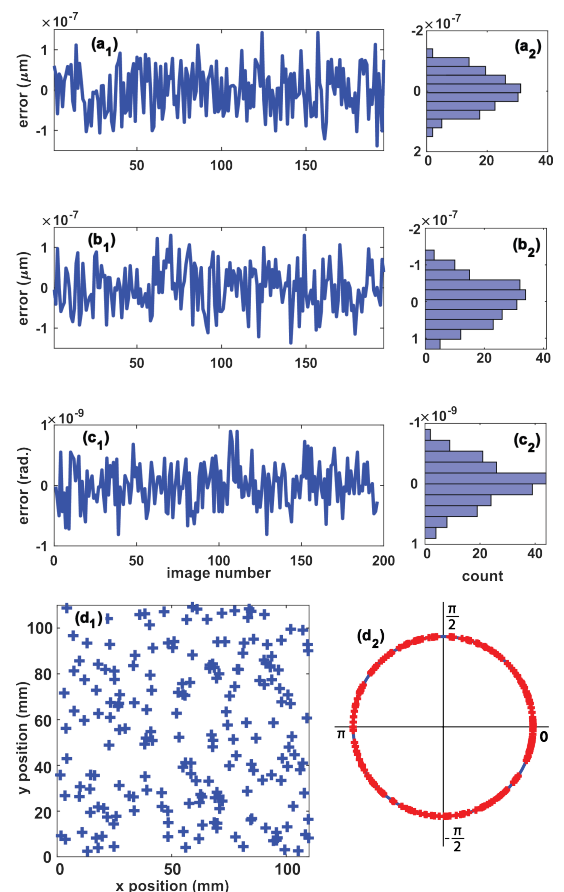

Fig. 5. Tested random positions $\left(a_{1}\right),\left(b_{1}\right),\left(c_{1}\right)$ : Reconstructed $(x, y, \theta)$ positions versus expected ones for a set of 200 random positions distributed on the whole target and $2 \pi \mathrm{rad} .\left(a_{2}\right),\left(b_{2}\right),\left(c_{2}\right)$ : corresponding histograms of deviations observed. $\left(d_{1}\right)$ shows the tested random positions on the target and $\left(d_{2}\right)$ shows the random angles used for the target simulation

TABLE II

STANDARD DEVIATIONS OF RESIDUAL ERRORS BETWEEN RECONSTRUCTED TARGET POSITIONS AND EXPECTED ONES. EXPLORED POSITIONS COVER THE WHOLE ENCODED AREA AND THE $(-\pi, \pi)$ INTERVAL.

\begin{tabular}{cc} 
axis & standard deviation \\
\hline$x$ & $5.78 \cdot 10^{-8} \mu \mathrm{m}$ \\
$y$ & $5.52 \cdot 10^{-8} \mu \mathrm{m}$ \\
$\theta$ & $3.40 \cdot 10^{-10} \mathrm{rad}$.
\end{tabular}

errors observed are summarized in Table $\Pi$ and result from the propagation of round-off error in the discrete Fourier transforms and further processing. However, the extremely small values obtained, in the range of $10^{-8} \mu \mathrm{m}$ and of $10^{-10} \mathrm{rad}$. demonstrate that, experimentally, the major obstacle to performances will result from setup imperfections and environmental disturbances.

\section{EXPERIMENTAL RESULTS}

\section{A. Large displacement measurements}

The performances of the proposed method were demonstrated using different setups and, to avoid frame rate limitations due to software, images were saved on the hard disk drive and then post-processed. Fig. 6 presents a picture of the 6 degrees of freedom Cartesian robot used for large displacement demonstration. The robot is composed of 3 prismatic and 3 rotational joints with integrated sensors and home assembled from SmartAct nanopositioning stages (more details in [23]). The robot allows preliminary focus adjustments over the whole encoded target surface as well as $10 \times 10 \mathrm{~cm}^{2}$ lateral displacements. The target is imaged onto a CMOS camera (UI-3280CP-M with a $3.45 \mu \mathrm{m}$ pixel size) through a $10 \times$ Mitutoyo lens (M plan Apo $10 \times$ N.A.0.28) and a imaging tube (Optem mini 1.5x 300 FL). With this hardware, a pixelic period of 19.5 pixels is expected and values retrieved by processing the recorded images software agree with this prediction. Fig. 7 a compares the $(x, y)$ positions reconstructed with the vision system to the control positions sent to the robot and used as references to validate the measurements. Unfortunately the displacement range allowed by the robot is smaller than the $11 \times 11 \mathrm{~cm}^{2}$ surface of the encoded target. Nevertheless, these results demonstrate the ability of the method to perform absolute position measurements over a range of $10 \times 10 \mathrm{~cm}^{2}$. Fig. 7 b shows the error observed between the reconstructed position and the control position sent to the robot. The line in red corresponds to a unique round-trip and its shape indicates that the error is strongly linked to the axis positions. These data could be used to calibrate the robot to improve its accuracy as it has been done in [24]. A video showing the position retrieving on a large scale $\mathrm{X}-\mathrm{Y}$ translation example is provided as supplementary material. 


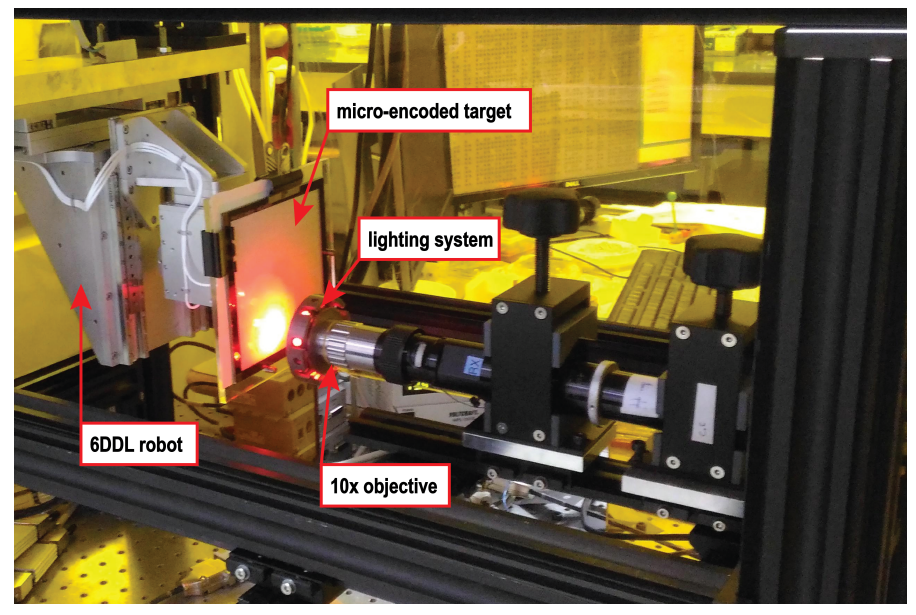

Fig. 6. Experimental setup used for large displacement measurements.
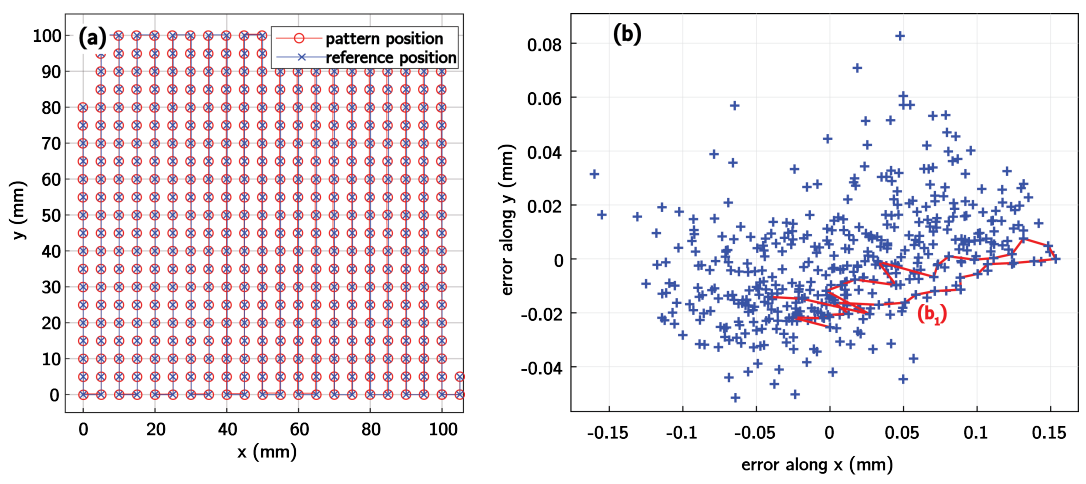

Fig. 7. (a) Reconstructed positions from a $10 \times 10 \mathrm{~cm}^{2}$ displacement experiment. (b) Differences observed between reconstructed positions and input commands send to the robot.

\section{B. Precision and linearity measurements}

The serial assembly of several single-axis actuators results in an increased uncertainty in the final position actually reached by the target. Therefore, we explored angular performances as well as ultimate lateral performances on dedicated setups involving a single actuator of interest. These supplementary experiments were carried out in a metrology room with temperature and humidity regulations. In Fig. 8 a $2 \times 2 \mathrm{~cm}^{2}$ piece of the target is fixed onto a Nano-MET10 (Mad City Labs) piezo-electric linear actuator with a $10 \mu \mathrm{m}$ range and an internal sensor resolution of $0.01 \mathrm{~nm}$. A complementary Smaract SR-2013 rotation stage with a closed loop resolution of $4.4 \cdot 10^{-7} \mathrm{rad}$. was used for angular measurements as shown in supplementary material.

Fig. 9 presents typical noise figures obtained while the target is kept static whereas Fig. $10 \mathrm{a}$ and $10 \mathrm{~b}$ present the linearity curves retrieved on a linear displacement of $9 \mu \mathrm{m}$ and on a rotation of $2 \pi \mathrm{rad}$. respectively. Table III summarizes the standard deviations of the reconstructed $(x, y, \theta)$ position with values on $x$ and $y$ smaller than one nanometer and of a few $10^{-6} \mathrm{rad}$. for in-plane orientation. Fig. 10 presents the deviation of the linearity data from the fitted lines for displacements and angles

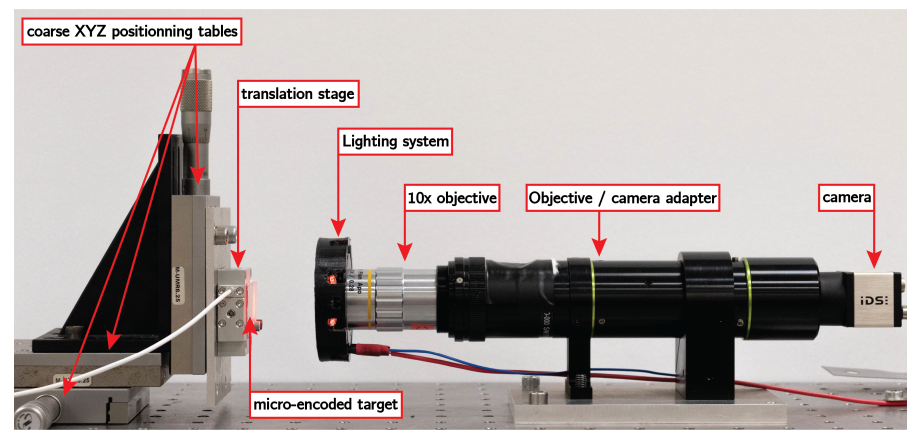

Fig. 8. Experimental setup used for linear displacement characterization. 

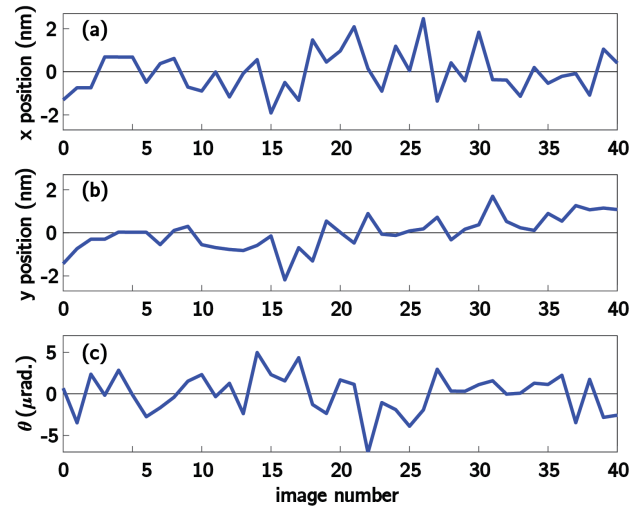

Fig. 9. Repetition of measurements with a static target.

TABLE III

PRECISION AND LINEARITY MEASUREMENTS (OVER EXCURSIONS OF $9 \mu \mathrm{M}$ FOR $x$ AND $y$ AND OF $2 \pi$ FOR $\theta$ )

\begin{tabular}{c|c|c|c} 
Axis & Precision $(\mathrm{std})$ & Linearity & Accuracy error \\
\hline$x$ & $0.96 \mathrm{~nm}$ & $0.4 \%$ & $1.15 \%$ \\
$y$ & $0.77 \mathrm{~nm}$ & - & - \\
$\theta$ & $4.07 \cdot 10^{-6} \mathrm{rad}$ & $0.00024 \%$ & $<0.004 \%$
\end{tabular}

respectively.

An excellent linearity is obtained in rotation with a coefficient of 0.999961 , meaning that the linear accuracy error is of a few $10^{-5}$. The maximum distance to the fitting line is of $8.59 \cdot 10^{-4} \mathrm{rad}$. and corresponds to $0.00024 \%$ of the $2 \pi$ range. Results are a bit less impressive in lateral position with a straight line coefficient of 1.011478 corresponding to a linear accuracy error of $1.15 \%$. This linear accuracy error questions the actual value of the target period that may slightly differ from the expected value of $9 \mu \mathrm{m}$. This issue will be addressed in a near future by calibrating the period value as well as its variations across the encoded area by means of a traceable and certified metrological inspection instrument. The maximum distance to the mean straight line is of $39 \mathrm{~nm}$ in displacement and corresponds to a linearity of $0.4 \%$ of the $9 \mu \mathrm{m}$ course performed in this test.
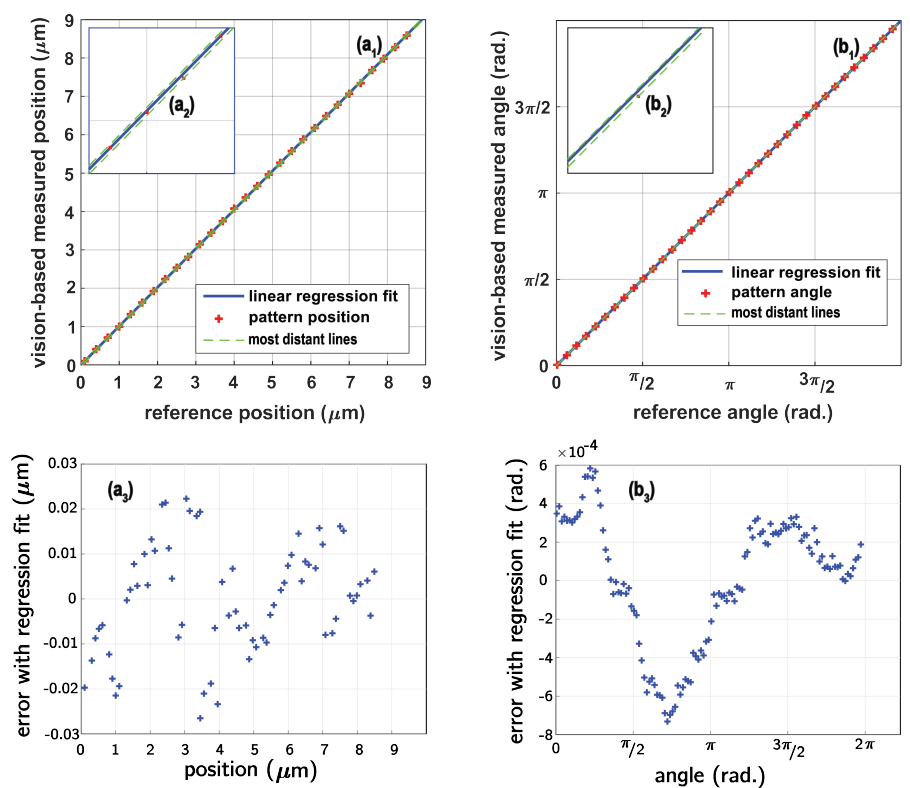

Fig. 10. Linearity tests: $\left(a_{1}\right)$ in translation $\widehat{x}=1.01148 x$ and $\left(b_{1}\right)$ rotation $\widehat{\theta}=0.999961 \theta$. Zooms in inserts $\left(a_{2}\right)$ and $\left(b_{2}\right)$ show the interval between most-distant lines. $\left(a_{3}\right)$ and $\left(b_{3}\right)$ show the distance between measured data and the regression fits. 

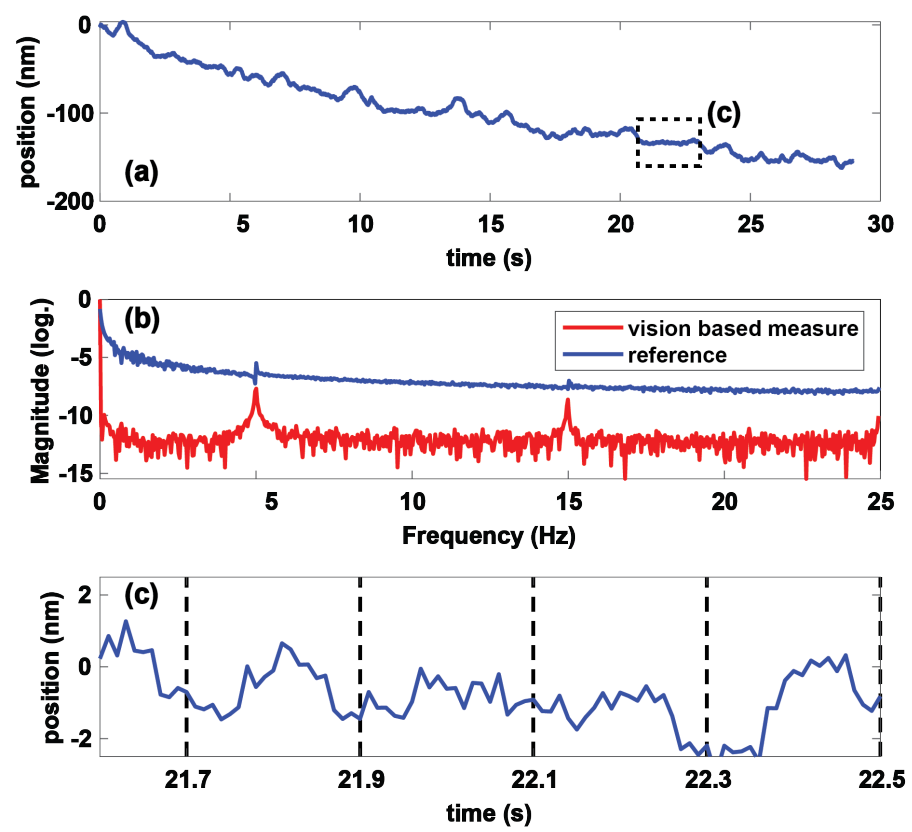

Fig. 11. (a) $1 \mathrm{~nm}$ lateral pulse $(5 \mathrm{~Hz}$ captured at $100 \mathrm{fps})$ sent during 1 second. (b) Fourier spectra of reconstructed and reference positions $(30 \mathrm{~s})$. (c) $1.7 \cdot 10^{-5} \mathrm{rad}$. pulse signal $(2 \mathrm{~Hz}$ at $20 \mathrm{fps})$ over a $2 \mathrm{~s}$ duration.

\section{Resolution estimations}

Resolution tests were also carried out and results obtained are presented in Figs. 11. For lateral resolution, we sent as input position to the piezoelectric actuator a square signal at a $5 \mathrm{~Hz}$ frequency with a $1 \mathrm{~nm}$ amplitude. Reconstructed positions are presented in Fig. 11. a. where the square shape of the signal is visible despite an important level of noise. This was confirmed by a Fourier transform applied to both the reconstructed positions and the data returned by the actuator internal sensor. Associated spectra are presented in Fig. 11. b where the expected spectral lobe is indeed present at the actual excitation frequency. The Fourier transform was applied to a $30 \mathrm{~s}$ sequence with some zones more noisy which explain the relatively small magnitude of this lobe in Fig. 11 b. As suggested by computer simulations, environmental disturbances, known to raise significant issues at the nanoscale, may constitute the primary cause of limitation of the resolution.

Fig. 11 c presents the results obtained in rotation while an input control signal with an amplitude of $1.7 \cdot 10^{-5} \mathrm{rad}$. at a frequency of $2 \mathrm{~Hz}$ was applied to the rotation actuator. The expected angular variation is clearly visible and the signal-to-noise observed suggests that even smaller angles could be detected. The precision of $4 \cdot 10^{-6} \mathrm{rad}$. previously estimated suggests that an improved resolution in this range should be achievable.

Unfortunately, the actuators used is unable to performs reliable changes smaller than these values and we could not explore experimentally the ultimate resolution of the method.

\section{Discussion of experimental performances}

This section aims to discuss the lateral resolution obtained that is limited at about one nanometer with this experimental setup. Uncertainty in measurement can be influenced by many different factors. We identified several sources of uncertainty in measurement that drastically increase the level of measurement errors. We first explored environmental instabilities as a major source of disturbances (vibrations, thermal changes, acoustic noise, etc.). In this aim, the series of 5 megapixel images that led to Fig. 9 was sub-divided into two sets of 2.5 megapixel images made of the left and right halves of every image respectively. Both series of images obtained were thus exposed to the same environmental disturbances that can be removed by operating a differential measurement between the two halves. By applying this procedure, the standard deviation of the distance between the centers of the half-images dropped from $0.96 \mathrm{~nm}$ to $0.24 \mathrm{~nm}$ in X and from $0.77 \mathrm{~nm}$ to $0.19 \mathrm{~nm}$ in $Y$. This result indicates that environmental disturbances are responsible for more than $93 \%$ of the total variance of errors. Fig. 12 presents the errors of reconstructed positions observed through this differential measurement procedure in regard to initial results (blue). The second factor of uncertainty that we investigated is the noise level in recorded images. The encoded target is made of holes etched on a thin chromium layer deposited on a glass plate that are originally aimed to serve as photomask for UV photolithography. Chromium is opaque to UV light but partially transparent to the red light used for experiments and suited to the spectral response of a CMOS camera. Combined with light scattered by the backside of the glass plate and illuminated in reflection, this configuration results in a low contrast and a high level of noise in recorded images. The typical standard deviation of the background of images is about 110 gray levels; i.e. $3 \%$ of the 3560 dynamics of the recorded images. The introduction of 

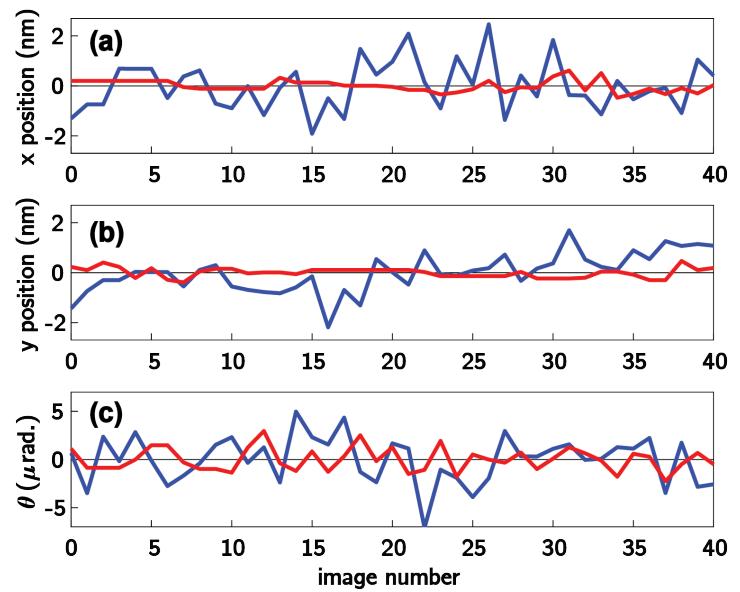

Fig. 12. Relative position discrepancies observed between the left and right halves of images (in red) in regard to the initial results of Fig 9 (in blue): (a) Along X; (b) Along Y (c) Along $\theta$.

a Gaussian noise with a magnitude of $3 \%$ of the dynamics in 100 synthesized images (kept at the same place as in case of Fig. 9) results in an error of $0.15 \mathrm{~nm}$ in $\mathrm{X}$ and $0.16 \mathrm{~nm}$ in $\mathrm{Y}$ which could explain up to $5 \%$ of the total experimental variance. The remaining sources of uncertainties such as the pattern fabrication, the optical distortions and the quantification are thus responsible for less than $2 \%$ of the variance of errors.

\section{CONCLUSION AND PROSPECTS}

This paper proposed a visual method for registering a micro-encoded target of large dimensions, i.e. $11 \times 11 \mathrm{~cm}^{2}$, that allows in-plane position measurements with a nanometer precision in displacement and of about $4 \cdot 10^{-6}$ rad. in orientation. The range-to-resolution ratio has been experimentally demonstrated to be at least $10^{8}$. Interferometry (IF) still provides a superior range-to-resolution ratio. However, IF is basically aimed to $1 \mathrm{D}$ linear measurements and multi-DoF measurements require cumbersome hardware. Furthermore, the measurement of angles with IF is indirect and restricted to short ranges. Therefore, the proposed method constitutes a relevant and convenient 3-DoF alternative to IF, especially for non-laboratory experiments, even if its ultimate range-to-resolution ratio remains smaller. As far as we know, there is no other 3-DoF measurement technique with such a resolution on such an extended measurement range.

The absolute binary encoding is based on LFSR sequences and allows unambiguous position recovery from a local image of less than 40 periods; i.e. $360 \times 360 \mu \mathrm{m}^{2}$ or $0.001 \%$ of the encoded area. The technique chosen to discriminate between 0 and 1 binary values does not introduce phase distortions and this avoids phase non-linearities that would be detrimental to performances.

The encoded target can be scaled for a different dimensional range as long as the magnification of the vision system is adjusted accordingly. The encoded target can be inserted in experimental setups to allow visual position control, if necessary after size reduction to meet actual needs and workspace capabilities. It can also be used to characterize or calibrate the motion of one to three degrees of freedom actuators. The method is self-calibrating and uses the design period as size reference that is available in any image of the target. However, this period is subject to some unknown degree of uncertainty and a metrological certification of its actual value and variations will be carried out in a near future. Major prospects concern the extension of the method to out-of-plane position measurement, for instance by using an interferometric imaging system to convert $\mathrm{z}$-axis motions into interferometric phase changes.

\section{ACKNOWLEDGMENT}

This work was supported by ROBOTEX (ANR-10-EQPX-44-01), by Cross-disciplinary Research (EIPHI) Graduate School (contract ANR-17-EURE-0002), the French Investissements d'Avenir program and the I-SITE Bourgogne Franche-Comté (BFC) project (contract ANR-15-IDEX-03). The encoded target was realized thanks to the RENATECH technological network and its FEMTO-ST facility MIMENTO. Authors acknowledge D. Raddenzati, L. Robert, M. Suarez and L. Gauthier-Manuel for technological and experimental assistance.

\section{REFERENCES}

[1] "The programs of manufacturing engineering," The National Institute of Standards and Technology, Tech. Rep., 2008.

[2] A. Assa and F. Janabi-Sharifi, "Virtual visual servoing for multicamera pose estimation," IEEE/ASME Transactions on Mechatronics, vol. 20, no. 2, pp. 789-798, 2014.

[3] T. Shu, S. Gharaaty, W. Xie, A. Joubair, and I. A. Bonev, "Dynamic path tracking of industrial robots with high accuracy using photogrammetry sensor," IEEE/ASME Transactions on Mechatronics, vol. 23, no. 3, pp. 1159-1170, 2018. 
[4] S. Liu, D. Xu, F. Liu, D. Zhang, and Z. Zhang, "Relative pose estimation for alignment of long cylindrical components based on microscopic vision," IEEE/ASME Transactions on Mechatronics, vol. 21, no. 3, pp. 1388-1398, 2015.

[5] C. Zhao, C. F. Cheung, and M. Liu, "Nanoscale measurement with pattern recognition of an ultra-precision diamond machined polar microstructure," Precision Engineering, vol. 56, pp. 156-163, 2019.

[6] J. Liu, Z. Gong, K. Tang, Z. Lu, and Y. Sun, "Locating end-effector tips in automated micromanipulation," in 2013 IEEE International Conference on Robotics and Automation. IEEE, 2013, pp. 1724-1729.

[7] H. K. Chu, J. K. Mills, and W. L. Cleghorn, "Dual-arm micromanipulation and handling of objects through visual images," in 2012 IEEE International Conference on Mechatronics and Automation. IEEE, 2012, pp. 813-818.

[8] E. Avci, C.-N. Nguyen, K. Ohara, M. Kojima, Y. Mae, and T. Arai, "Towards high-speed automated micromanipulation," in 2013 IEEE International Conference on Robotics and Automation. IEEE, 2013, pp. 1718-1723.

[9] W. Shang, H. Lu, W. Wan, T. Fukuda, and Y. Shen, "Vision-based nano robotic system for high-throughput non-embedded cell cutting," Scientific reports, vol. 6, p. 22534, 2016.

[10] H. Li, B. Zhu, Z. Chen, and X. Zhang, "Realtime in-plane displacements tracking of the precision positioning stage based on computer micro-vision," Mechanical Systems and Signal Processing, vol. 124, pp. 111-123, 2019.

[11] W. Huang, C. Ma, and Y. Chen, "Displacement measurement with nanoscale resolution using a coded micro-mark and digital image correlation," Optical Engineering, vol. 53, no. 12, p. 124103, 2014

[12] G. Bomarito, J. Hochhalter, T. Ruggles, and A. Cannon, "Increasing accuracy and precision of digital image correlation through pattern optimization," Optics and Lasers in Engineering, vol. 91, pp. 73-85, 2017.

[13] J. Kokorian, F. Buja, and W. M. van Spengen, "In-plane displacement detection with picometer accuracy on a conventional microscope," Journal of Microelectromechanical Systems, vol. 24, no. 3, pp. 618-625, 2014.

[14] C. Yamahata, E. Sarajlic, G. J. Krijnen, and M. A. Gijs, "Subnanometer translation of microelectromechanical systems measured by discrete fourier analysis of ccd images," Journal of Microelectromechanical Systems, vol. 19, no. 5, pp. 1273-1275, 2010.

[15] V. Guelpa, P. Sandoz, M. A. Vergara, C. Clévy, N. Le Fort-Piat, and G. J. Laurent, "2d visual micro-position measurement based on intertwined twin-scale patterns," Sensors and Actuators A: Physical, vol. 248, pp. 272-280, 2016.

[16] V. Guelpa, G. J. Laurent, P. Sandoz, J. A. Galeano-Zea, and C. Clévy, "Subpixelic measurement of large 1D displacements: Principle, processing algorithms, performances and software," Sensors (Switzerland), vol. 14, no. 3, pp. 5056-5073, mar 2014.

[17] H. Sugiura, S. Sakuma, M. Kaneko, and F. Arai, "On-chip method to measure mechanical characteristics of a single cell by using moiré fringe," Micromachines, vol. 6, no. 6, pp. 660-673, 2015.

[18] J. Z. Galeano, P. Sandoz, E. Gaiffe, S. Launay, L. Robert, M. Jacquot, F. Hirchaud, J.-L. Pretet, and C. Mougin, "Position-referenced microscopy for live cell culture monitoring." Biomedical optics express, vol. 2, no. 5, pp. 1307-1318, 2011.

[19] J. Stevenson and J. Jordan, "Absolute position measurement using optical detection of coded patterns," Journal of Physics E: Scientific Instruments, vol. 21 , no. 12 , p. 1140,1988

[20] P. Masa, E. Franzi, and C. Urban, "Nanometric Resolution Absolute Position Encoder," in Proc. 13th European Space Mechanisms and Tribology Symposium, 2009

[21] J.-A. Kim, J. W. Kim, C.-S. Kang, J. Jin, and T. B. Eom, "Note: Nonlinearity error compensated absolute planar position measurement using a twodimensional phase-encoded binary grating," Review of Scientific Instruments, vol. 84, no. 5, p. 056102, 2013

[22] J. A. Galeano-Zea, P. Sandoz, and L. Robert, "Position encryption of extended surfaces for subpixel localization of small-sized fields of observation," in 2009 International Symposium on Optomechatronic Technologies. IEEE, 2009, pp. 22-27.

[23] J.-Y. Rauch, O. Lehmann, P. Rougeot, J. Abadie, J. Agnus, and M. A. Suarez, "Smallest microhouse in the world, assembled on the facet of an optical fiber by origami and welded in the $\mu$ robotex nanofactory," Journal of Vacuum Science \& Technology A: Vacuum, Surfaces, and Films, vol. 36, no. 4, p. 041601, 2018.

[24] N. Tan, C. Clévy, G. J. Laurent, P. Sandoz, and N. Chaillet, "Accuracy quantification and improvement of serial micropositioning robots for in-plane motions," IEEE Transactions on robotics, vol. 31, no. 6, pp. 1497-1507, Dec. 2015. 